\title{
Management of Pediatric Nail Psoriasis
}

\author{
Kerasia-Maria Plachouri, MD, PhD; Francesk Mulita, MD, PhD; Sophia Georgiou, MD, PhD
}

\section{PRACTICE POINTS}

- No clinical trials assessing the management of pediatric nail psoriasis currently are present in the literature. Limited information on the treatment of pediatric nail psoriasis exists, mostly in the form of small case series and case reports.

- As more agents are approved for on-label use in plaque psoriasis in pediatric patients, gradually more real-life data on their efficacy for nail psoriasis in children are expected to come to light.

Nail psoriasis is a condition that can affect children and adolescents. It often is refractory to treatment. Data on its management in the pediatric population are limited. This article aims to summarize existing information-small case series and case reports-on the successful therapeutic approaches for nail psoriasis in children. As more agents are approved for on-label use in plaque psoriasis in pediatric patients, gradually more real-life data on their efficacy for nail psoriasis in children are expected to come to light.

Cutis. 2021;108:292-294.

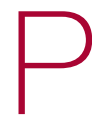

ediatric nail psoriasis is a condition that has not been extensively studied. The prevalence of nail alterations in pediatric patients with psoriasis varies among different studies, ranging from $17 \%$ to $39.2 \% .{ }^{1}$ Nail pitting, onycholysis associated with subungual hyperkeratosis, paronychia, and pachyonychia are the most frequent features of psoriatic nail involvement in children., ${ }^{2,3}$ The management of nail psoriasis in children and adolescents is critical due to the quality-of-life impact, from potential functional impairment issues to the obvious cosmetic problems, which can aggravate the psychologic distress and social embarrassment of patients with psoriasis. Despite the emergence of modern potent systemic agents to treat chronic plaque psoriasis, nail psoriasis often is refractory to treatment. ${ }^{4}$ Coupled with the limited on-label options for psoriasis treatment in children, the management of nail psoriasis in this special patient group constitutes an even greater therapeutic challenge. This report aims to summarize the limited existing data on the successful management of nail psoriasis in the pediatric population.

\section{Reviewing the Literature on Nail Psoriasis}

We conducted a search of PubMed articles indexed for MEDLINE, Embase, and Scopus using the following Medical Subject Headings key terms: nail psoriasis and children, juvenile, pediatric. Additional articles were identified from the reference lists of the retrieved articles and citations. Our search included reports in the English language published from 2000 to 2019. The selection process included the following 2 steps: screening of the titles and abstracts, followed by evaluation of the selected fulltext articles.

\section{Topical Treatments for Nail Psoriasis}

Because most systemic antipsoriatic treatments that can be administered in adult patients have not yet been granted an official license for administration in children, topical treatments are considered by many physicians as the preferred first-line therapy for psoriatic nail involvement in pediatric patients. ${ }^{5,6}$ However, only scarce data are available in the literature concerning the successful use of local agents in pediatric patients with psoriasis.

From the University General Hospital of Patras, Greece. Drs. Plachouri and Georgiou are from the Department of Dermatology, and Dr. Mulita is from the Department of General Surgery.

The authors report no conflict of interest.

The eFigure is available in the Appendix online at www.mdedge.com/dermatology.

Correspondence: Kearse-Maria Plachouri, MD, PhD, University General Hospital of Patras, Rio 265 04, Greece (kerasia.plachouri@hotmail.com). doi: 10.12788 /cutis.0386 
The main limitation of local treatments relates mostly to their impaired penetration into the affected area (nails). To optimize drug penetration, some authors suggest the use of potent keratolytic topical preparations to reduce the nail volume and facilitate drug absorption. ${ }^{7}$ A popular suggestion is trimming the onycholytic nail plate followed by $40 \%$ urea avulsion to treat subungual hyperkeratosis 8 or simply the use of occlusive $40 \%$ urea in petroleum jelly. ${ }^{9}$ Other approaches include clipping the onycholytic nail over the diseased nail bed or processing the nail plate through grinding or even drilling holes with the use of mechanical burrs or ablative lasers to enhance the penetration of the topical agent. ${ }^{\text {? }}$

A frequent approach in pediatric patients is clipping the detached nails combined with daily application of calcipotriene (calcipotriol) and steroids, such as betamethasone dipropionate., ${ }^{5,8}$ Reports on the use of regimens with clobetasol propionate ointment 0.05\% under occlusion, with or without the concomitant use of calcipotriol solution $0.005 \%$, also are present in the literature but not always with satisfactory results. ${ }^{10,11}$ Another successfully administered topical steroid is mometasone furoate cream $0.1 \% .^{12}$ Although the use of intralesional triamcinolone acetonide also has demonstrated encouraging outcomes in isolated reports, ${ }^{13}$ associated adverse events, such as pain and hematomas, can result in tolerability issues for pediatric patients. ${ }^{7}$

Piraccini et $\mathrm{al}^{14}$ described the case of an 8 -year-old patient with pustular nail psoriasis who showed improvement within 3 to 6 months of treatment with topical calcipotriol $5 \mu \mathrm{g} / \mathrm{g}$ as monotherapy applied to the nail and periungual tissues twice daily. Another approach, described by Diluvio et $\mathrm{al}^{15}$ is the use of tazarotene gel $0.05 \%$ applied once daily to the affected nail plates, nail folds, and periungual skin without occlusion. In a 6-yearold patient with isolated nail psoriasis, this treatment regimen demonstrated notable improvement within 8 weeks. ${ }^{15}$

\section{Systemic Treatments for Nail Psoriasis}

Data on the successful administration of systemic agents in pediatric patients also are extremely scarce. Due to the lack of clinical trials, everyday practice is mostly based on isolated case series and case reports.

Methotrexate- Lee $^{11}$ described the case of an 11-yearold girl with severe, symptomatic, 20-nail psoriatic onychodystrophy who showed a complete response to oral methotrexate $5 \mathrm{mg} / \mathrm{wk}$ after topical clobetasol propionate and calcipotriol failed. Improvement was seen as early as 4 weeks after therapy initiation, and complete resolution of the lesions was documented after 9 and 13 months of methotrexate therapy for the fingers and toes, respectively. ${ }^{11}$ The successful use of methotrexate in the improvement of psoriatic nail dystrophy in a pediatric patient also was documented by Teran et al. ${ }^{16}$ In this case, a 9-year-old girl with erythrodermic psoriasis, psoriatic arthritis, and severe onychodystrophy showed notable amelioration of all psoriatic manifestations, including the nail findings, with systemic methotrexate therapy (dose not specified).$^{16}$ Notably, the authors reported that the improvement of onychodystrophy occurred with considerable delay compared to the other psoriatic lesions, ${ }^{16}$ indicating the already-known refractoriness of nail psoriasis to the various therapeutic attempts. . $^{9-15}$

Acitretin-Another agent that has been linked with partial improvement of acrodermatitis continua of Hallopeau (ACH)-associated onychodystrophy is acitretin. In a case series of 15 pediatric patients with pustular psoriasis, a 5-year-old boy with severe nail involvement presented with partial amelioration of nail changes with acitretin within the first 6 weeks of treatment using the following regimen: initial dosage of $0.8 \mathrm{mg} / \mathrm{kg} / \mathrm{d}$ for 6 weeks, followed by $0.3 \mathrm{mg} / \mathrm{kg} / \mathrm{d}$ for 4 weeks. ${ }^{17}$

Biologics-The emerging use of biologics in pediatric psoriasis also has brought important advances in the successful management of nail psoriasis in children and adolescents. ${ }^{18-21}$ Wells et $\mathrm{al}^{18}$ presented the case of an 8-year-old girl with nail psoriasis, psoriatic arthritis, and plaque psoriasis who showed complete resolution of all psoriatic manifestations, including nail involvement, within 3 months of treatment with secukinumab $150 \mathrm{mg}$ subcutaneously every 4 weeks. Prior failed treatments included various systemic agents (ie, subcutaneous methotrexate $20 \mathrm{mg} / \mathrm{m}^{2}$, etanercept $0.8 \mathrm{mg} / \mathrm{kg}$ weekly, adalimumab $40 \mathrm{mg}$ every 2 weeks) as well as topical agents (ie, urea, tazarotene, corticosteroids) and intralesional triamcinolone. ${ }^{18}$

Infliximab also has been successfully used for pediatric nail psoriasis. Watabe et $\mathrm{al}^{19}$ presented the case of an 8 -year-old girl with psoriatic onychodystrophy in addition to psoriatic onycho-pachydermo-periostitis. Prior therapy with adalimumab $20 \mathrm{mg}$ every other week combined with methotrexate $10 \mathrm{mg}$ weekly failed. She experienced notable amelioration of the nail dystrophy within 3 months of using a combination of infliximab and methotrexate (infliximab $5 \mathrm{mg} / \mathrm{kg}$ intravenously on weeks 0,2 , and 6 , and every 8 weeks thereafter; methotrexate $10 \mathrm{mg} / \mathrm{wk}) .{ }^{19}$

Cases in which infliximab has resulted in rapid yet only transient restoration of psoriatic onychodystrophy also are present in the literature. Pereira et $\mathrm{al}^{20}$ reported that a 3-year-old patient with severe 20-digit onychodystrophy in addition to pustular psoriasis had complete resolution of nail lesions within 2 weeks of treatment with infliximab $(5 \mathrm{mg} / \mathrm{kg}$ at weeks 0,2 , and 6 , and then every 7 weeks thereafter), which was sustained over the course of 1 year. The therapy had to be discontinued because of exacerbation of the cutaneous symptoms; thereafter, etanercept was initiated. Although the patient noted major improvement of all skin lesions under etanercept, only moderate amelioration of the psoriatic nail lesions was demonstrated. ${ }^{20}$

Dini et $\mathrm{al}^{21}$ described a 9-year-old girl with severe $\mathrm{ACH}$-associated psoriatic onychodystrophy who showed complete clearance of all lesions within 8 weeks of treatment with adalimumab (initially $80 \mathrm{mg}$, followed by 
$40 \mathrm{mg}$ after 1 week and then $40 \mathrm{mg}$ every other week). Prior treatment with potent topical corticosteroids, cyclosporine ( $3 \mathrm{mg} / \mathrm{kg} / \mathrm{d}$ for 6 months), and etanercept $\left(0.4 \mathrm{mg} / \mathrm{kg}\right.$ twice weekly for 3 months) was ineffective. ${ }^{21}$

Phototherapy-Other systemic agents with reported satisfactory outcomes in the treatment of psoriatic onychodystrophy include thalidomide combined with UVB phototherapy. Kiszewski et $\mathrm{al}^{22}$ described a 2-year-old patient with $\mathrm{ACH}$ and severe 19-digit onychodystrophy. Prior failed therapies included occluded clobetasol ointment $0.05 \%$, occluded pimecrolimus $0.1 \%$, and systemic methotrexate, while systemic acitretin $(0.8 \mathrm{mg} / \mathrm{kg} / \mathrm{d})$ resulted in elevated cholesterol levels and therefore had to be interrupted. Improvement was seen 2 months after the initiation of a combined broadband UVB and thalidomide (50 mg/d) treatment, with no documented relapses after discontinuation of therapy. ${ }^{22}$

Narrowband UVB (311 nm) also has been used as monotherapy for $\mathrm{ACH}$-associated onychodystrophy, as demonstrated by Bordignon et $\mathrm{al}^{23}$ They reported a 9 -year-old patient who showed partial improvement of isolated onychodystrophy of the fourth nail plate of the left hand after 36 sessions of narrowband UVB using a 311-nm filtering handpiece with a square spot size of $19 \times 19 \mathrm{~mm}^{23}$

\section{Conclusion}

Nail psoriasis constitutes a type of psoriasis that is not only refractory to most treatments but is accompanied by substantial psychological and occasionally functional burden for the affected individuals. ${ }^{24}$ Data concerning therapeutic options in the pediatric population are extremely limited, and therefore the everyday practice often involves administration of off-label medications, which can constitute a dilemma for many physicians, especially for safety. ${ }^{10}$ We suggest a simple therapeutic algorithm for the management of pediatric nail psoriasis based on the summarized data that are currently available in the literature. This algorithm is shown in the eFigure.

As progressively more agents-especially biologicsreceive approval for use in plaque psoriasis in pediatric patients, ${ }^{25}$ it is expected that gradually more real-life data on their side efficacy for plaque psoriasis of the nails in children also will come to light. Furthermore, their on-label use in pediatric psoriasis patients will facilitate further relevant clinical trials to this target group so that the potential of these medications in the management of nail psoriasis can be fully explored.

\section{REFERENCES}

1. Uber M, Carvalho VO, Abagge KT, et al. Clinical features and nail clippings in 52 children with psoriasis. Pediatr Dermatol. 2018;35:202-207.

2. Pourchot D, Bodemer C, Phan A, et al. Nail psoriasis: a systematic evaluation in 313 children with psoriasis. Pediatr Dermatol. 2017;34:58-63.
3. Piraccini BM, Triantafyllopoulou I, Prevezas C, et al. Nail psoriasis in children: common or uncommon? results from a 10-year double-center study. Skin Appendage Disord. 2015;1:43-48.

4. Baran R. The burden of nail psoriasis: an introduction. Dermatology. 2010;221(suppl 1):1-5.

5. Richert B, André J. Nail disorders in children: diagnosis and management. Am J Clin Dermatol. 2011;12:101-112.

6. Trüeb RM. Therapies for childhood psoriasis. Curr Probl Dermatol. 2009;38:137-159.

7. Haneke E. Nail psoriasis: clinical features, pathogenesis, differential diagnoses, and management. Psoriasis (Auckl). 2017;7:51-63.

8. Piraccini BM, Starace M. Nail disorders in infants and children. Curr Opin Pediatr. 2014;26:440-445.

9. Duran-McKinster C, Ortiz-Solis D, Granados J, et al. Juvenile psoriatic arthritis with nail psoriasis in the absence of cutaneous lesions. Int J Dermatol. 2000;39:32-35.

10. Holzberg M, Ruben BS, Baran R. Psoriasis restricted to the nail in a 7-year-old child. should biologics be an appropriate treatment modality when considering quality of life? J Eur Acad Dermatol Venereol. 2014;28:668-670.

11. Lee JY. Severe 20-nail psoriasis successfully treated by low dose methotrexate. Dermatol Online J. 2009;15:8

12. Liao YC, Lee JY. Psoriasis in a 3-month-old infant with Kawasaki disease. Dermatol Online J. 2009;15:10.

13. Khoo BP, Giam YC. A pilot study on the role of intralesional triamcinolone acetonide in the treatment of pitted nails in children. Singapore Med J. 2000;41:66-68.

14. Piraccini BM, Tosti A, Iorizzo M, et al. Pustular psoriasis of the nails: treatment and long-term follow-up of 46 patients. $\mathrm{Br} J$ Dermatol. 2001;144:1000-1005.

15. Diluvio L, Campione E, Paternò EJ, et al. Childhood nail psoriasis: a useful treatment with tazarotene 0.05\%. Pediatr Dermatol. 2007; 24:332-333

16. Teran CG, Teran-Escalera CN, Balderrama C. A severe case of erythrodermic psoriasis associated with advanced nail and joint manifestations: a case report. J Med Case Rep. 2010;4:179.

17. Chen P, Li C, Xue R, et al. Efficacy and safety of acitretin monotherapy in children with pustular psoriasis: results from 15 cases and a literature review. J Dermatolog Treat. 2018;29:353-363.

18. Wells LE, Evans T, Hilton R, et al. Use of secukinumab in a pediatric patient leads to significant improvement in nail psoriasis and psoriatic arthritis. Pediatr Dermatol. 2019;36:384-385.

19. Watabe D, Endoh K, Maeda F, et al. Childhood-onset psoriatic onycho-pachydermo-periostitis treated successfully with infliximab. Eur J Dermatol. 2015;25:506-508.

20. Pereira TM, Vieira AP, Fernandes JC, et al. Anti-TNF-alpha therapy in childhood pustular psoriasis. Dermatology. 2006;213:350-352.

21. Dini V, Barbanera S, Romanelli M. Efficacy of adalimumab for the treatment of refractory paediatric acrodermatitis continua of Hallopeau. Acta Derm Venereol. 2013;93:588-589.

22. Kiszewski AE, DeVilla D, Scheibel I, et al. An infant with acrodermatitis continua of Hallopeau: successful treatment with thalidomide and UVB therapy. Pediatr Dermatol. 2009;26:105-106.

23. Bordignon M, Zattra E, Albertin C, et al. Successful treatment of a 9-year-old boy affected by acrodermatitis continua of Hallopeau with targeted ultraviolet B narrow-band phototherapy. Photodermatol Photoimmunol Photomed. 2010;26:41-43.

24. Fabroni C, Gori A, Troiano $\mathrm{M}$, et al. Infliximab efficacy in nail psoriasis. a retrospective study in 48 patients. J Eur Acad Dermatol Venereol. 2011;25:549-553.

25. Lilly's Taltz® (ixekizumab) receives U.S. FDA approval for the treatment of pediatric patients with moderate to severe plaque psoriasis. Eli Lilly and Company. March 30, 2020. Accessed September 24, 2021. https://investor.lilly.com/news-releases/news-release-details /lillys-taltzr-ixekizumab-receives-us-fda-approval-treatment-1 


\section{APPENDIX}
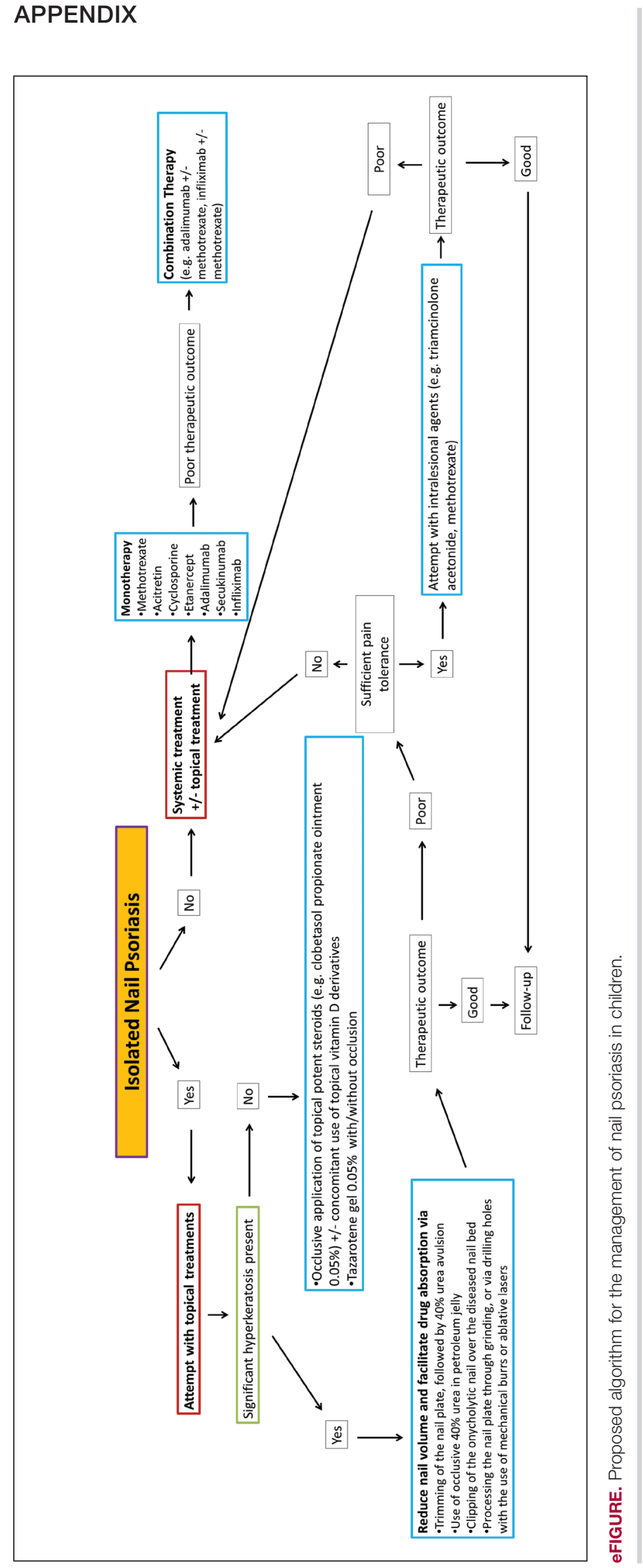